\title{
Correspondence
}

\section{Demonstration of a shift towards penicillin resistance in the Streptococcus uberis population}

Penicillin resistance has been particularly well studied in Streptococcus pneumoniae (Denapaite et al., 2007; Hoban et al., 2005). In this organism, resistance frequently occurs through modifications of the penicillin-binding proteins (PBPs) and/or the generation of mosaic PBPs, leading to a decreased affinity for the drug (Nagai et al., 2002). Among clinically relevant streptococci, S. pneumoniae primarily infects humans, while the environmental Streptococcus uberis principally colonizes cattle, in which it is responsible for about one third of cases of clinical and subclinical mastitis (Botrel et al., 2010; Bradley et al., 2007), a type of disease causing major economic loss in the dairy industry worldwide. Currently, penicillin remains one of the first-line antibiotics for the treatment of such pathologies but, in contrast to pneumococci and despite several decades of widespread use, no field case of true penicillin-resistant $S$. uberis [MIC $>16 \mathrm{mg} \mathrm{l}^{-1}$, according to the CASFM (Comité de l'Antibiogramme de la Société Française de Microbiologie) Antibiogram Committee of the French Society for Microbiology] has been described yet. Furthermore, it is still commonly believed that $S$. uberis and other Streptococcus spp. implicated in animal intramammary infections are susceptible to this drug. However, isolates presenting decreased susceptibility (MIC of 0.25 to $0.5 \mathrm{mg} \mathrm{l}^{-1}$ ) are regularly reported to our laboratory, and a recent study demonstrated the capacity of S. uberis to develop penicillin resistance through a PBP-mediated mechanism similar to $S$. pneumoniae and the other streptococci studied so far (Haenni et al., 2010).

At present, the official criteria for the MIC determination of penicillin-resistant $S$. pneumoniae (sensitive, $<0.06 \mathrm{mg} \mathrm{l}^{-1}$; resistant, $\geqslant 2 \mathrm{mg} \mathrm{l}^{-1}$ ) are coherent between the European Committee on Antimicrobial Susceptibility Testing (http://www.eucast.org), the American Clinical and Laboratory Standards
Institute and the French CA-SFM (CASFM, 2008; CLSI, 2008). On the contrary, criteria for non-S. pneumoniae streptococci are not harmonized and the classification of $S$. uberis itself is problematic. This bacterium was formerly associated to the viridans group because of its $\alpha$-haemolysis, but is now considered as a member of the pyogenic group with a close phylogenetic link to Streptococcus pyogenes, which is apparently incapable of acquiring penicillin resistance in vivo (Perez-Trallero et al., 2001). In France, the CA-SFM recommends that penicillin resistance is suspected for all non-pneumococcal streptococci isolated from animals when a decreased susceptibility to $5 \mu \mathrm{g}$ oxacillin is detected by disc diffusion (resistant if $<21 \mathrm{~mm}$ ). Since it was recently shown that $S$. uberis can develop resistance to penicillin in vitro (Haenni et al., 2010), and since isolates presenting decreased susceptibility have already been reported (Guerin-Faublee et al., 2002; Mevius et al., 2007; Rossitto et al., 2002), this work intended to further characterize field isolates classified as either oxacillin resistant or oxacillin sensitive, in order to get a better insight of the potential population shift towards penicillin resistance in $S$. uberis.

A total of 185 S. uberis strains isolated from bovine mastitis and identified by species-specific PCRs (Riffon et al., 2001) was included in this study. A total of 90 strains were considered as oxacillin resistant $\left(\mathrm{oxa}^{\mathrm{R}}\right)$ with disc diffusion diameters ranging between 15 and $20 \mathrm{~mm}$, whereas 95 were oxacillin sensitive ( $\mathrm{oxa}^{\mathrm{s}}$ ) with diameters ranging from 21 to $40 \mathrm{~mm}$. MICs to oxacillin, penicillin, amoxicillin and ampicillin were then determined by the agar dilution method on MullerHinton agar containing $5 \%$ sheep blood (Bio-Rad), by twofold dilutions ranging from 0.002 to $8 \mathrm{mg} \mathrm{l}^{-1}$. All isolates were tested according to the recommendations of the CA-SFM (2008).

As presented in Fig. 1, the 90 oxa $^{\mathrm{S}}$ strains detected by disc diffusion were confirmed as negative by the agar dilution method. On the contrary, only 2 strains out of 95 oxa ${ }^{\mathrm{R}}$ were considered as resistant, despite the fact that both populations differed significantly $(P<0.01$, Student's $t$-test $)$.

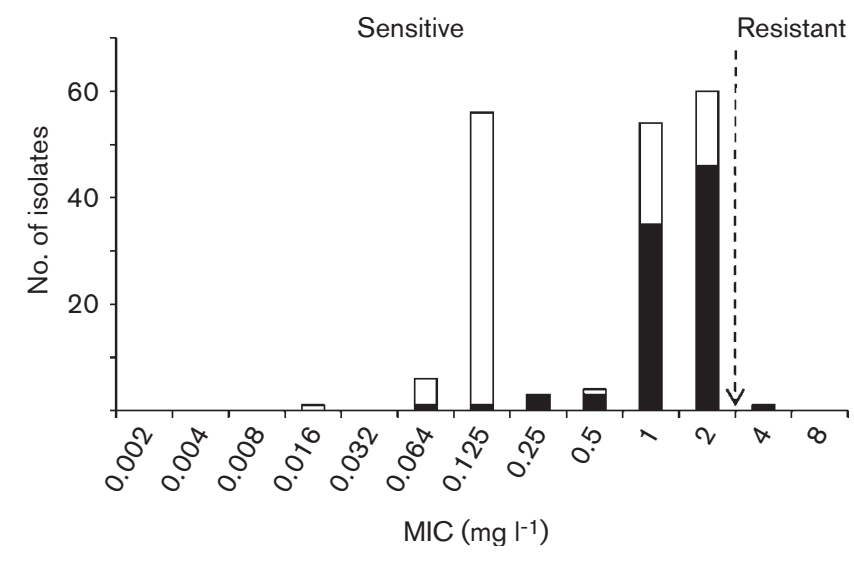

Fig. 1. Distribution of the $\mathrm{MIC}$ values $\left(\mathrm{mg} \mathrm{I}^{-1}\right)$ for oxacillin. Results of the population described as oxacillin sensitive by disc diffusion are indicated by white bars, and those described as oxacillin resistant by disc diffusion are indicated by black bars. The arrow indicates the breakpoint between sensitive and resistant isolates. 
This discrepancy proves that the oxacillin disc diffusion test is a poor indicator of clinical resistance and should not have been used as a predictor of the therapeutic outcome.

Similar results were observed for penicillin and amoxicillin (Fig. 2a, b). Indeed, the bimodal distributions demonstrated a shift in the population, yet all isolates were classified as sensitive according to the CA-
SFM. For ampicillin (Fig. 2c), there was a step-by-step evolution towards decreased resistance, and $46 \mathrm{oxa}^{\mathrm{R}}$ strains $(48 \%)$ were considered as having intermediate resistance by the agar dilution method. Thus, for this particular antibiotic, the population shift towards resistance was congruent with a modification in the resistance pattern. Therefore, the oxacillin disc result can be used as a marker of (a)

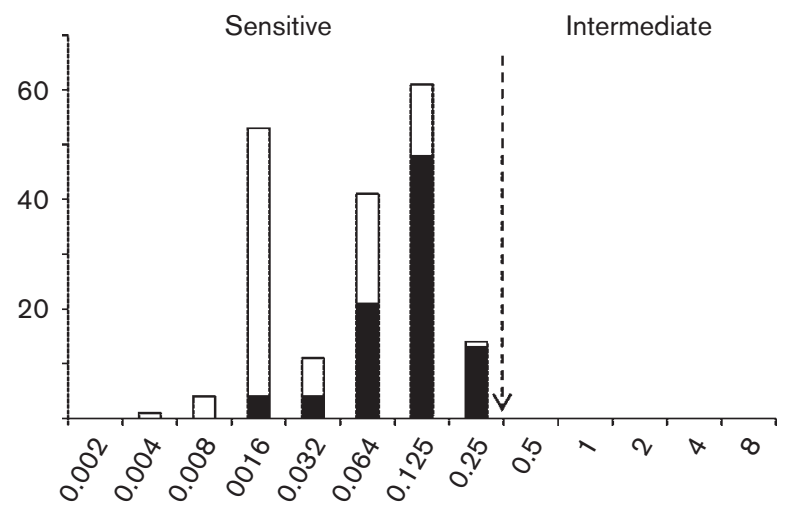

(b)

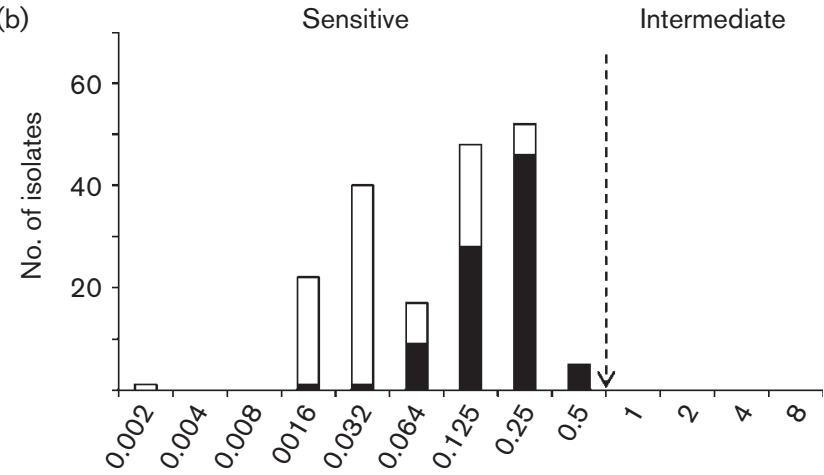

(c)

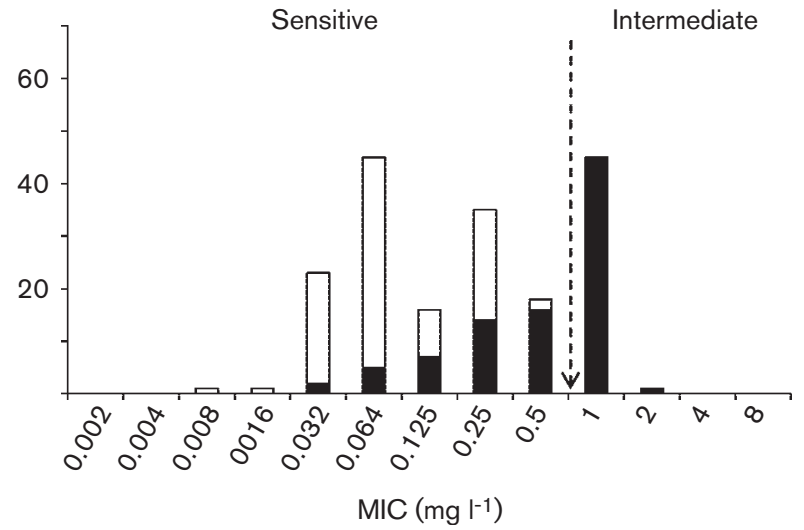

Fig. 2. Distribution of the MIC values $\left(\mathrm{mg} \mathrm{I}^{-1}\right.$ ) to penicillin (a), amoxicillin (b) and ampicillin (c). Results of the population described as oxacillin sensitive by disc diffusion are indicated by white bars, and those of the oxacillin resistant population by disc diffusion are indicated by black bars. The arrows indicate the breakpoints between sensitive and intermediate isolates. epidemiological evolution, independently of any clinical consideration.

The reality of this shift can also be inferred from our recent data on the molecular mechanisms of penicillin resistance in $S$. uberis (Haenni et al., 2010). Indeed, in that study, the presence of penicillin-resistanceassociated mutations (including the prototypic $\mathrm{Q}_{554} \mathrm{E}$ substitution in the PBP2X) was systematically detected in $S$. uberis presenting an MIC above $0.12 \mathrm{mg} \mathrm{l}^{-1}$ - in both laboratory-evolved mutants and field isolates - whereas these mutations were absent in the isolates presenting a MIC of $0.06 \mathrm{mg} \mathrm{l}^{-1}$. Therefore, while still considered as sensitive according to the French clinical breakpoints, isolates presenting an MIC $\geqslant 0.12 \mathrm{mg} \mathrm{l}^{-1}$ already display resistance mechanisms to $\beta$ lactams. Consequently, these isolates cannot be considered anymore as epidemiologically representative of a wildtype sensitive population and, moreover, contribute to an animal reservoir of potentially exchangeable PBP-resistant determinants.

In conclusion, this study confirms that a subpopulation of $S$. uberis field isolates shifted towards penicillin resistance, despite the fact that these isolates were still all considered as sensitive to this drug. These results raise the question of the pertinence of using penicillins to treat streptococcal bovine mastitis, a consideration in tight relation with the rapidity with which this population shift might evolve. However, only a combination of field and scientific observations might help tackle this difficult issue. Indeed, on the one hand, veterinary habits tend to indicate that penicillins are still widely and successfully used in the case of intramammary infections and, on the other hand, the slow development of resistance-associated mutations in vitro, as well as the apparent absence of mosaic PBPs in mastitis isolates (Haenni et al., 2010), does not argue for a rapid and broad emergence of resistance, contrary to pneumococci in human medicine. Nevertheless, the reality of these phenotypic and molecular changes in $S$. uberis must not be underestimated, especially as similar trends were recently reported in Streptococcus agalactiae, a pathogen that is associated both with animal and severe human diseases 
(Kimura et al., 2008). Thus, taken together, these results underline the need to perform regular surveys on $S$. uberis field isolates, first to monitor the evolution of this population shift, and second to avoid a wider spread of penicillin-resistant streptococci in veterinary medicine.

\section{Marisa Haenni, Estelle Saras and Jean-Yves Madec}

Agence Française de Sécurité Sanitaire des Aliments (AFSSA), Unité

Bactériologie et Virulence Bactériennes, 31 Avenue Tony Garnier, 69364 Lyon,

France

Correspondence: Jean-Yves Madec (jy.madec@afssa.fr)

Botrel, M.-A., Haenni, M., Morignat, E., Sulpice, P., Madec, J.-Y. \& Calavas, D. (2010).

Distribution and antimicrobial resistance of clinical and subclinical mastitis pathogens in dairy cows in Rhône-Alpes, France. Foodborne Pathog Dis 7, 479-487.

Bradley, A. J., Leach, K. A., Breen, J. E., Green, L. E. \& Green, M. J. (2007). Survey of the incidence and aetiology of mastitis on dairy farms in England and Wales. Vet Rec 160, 253258.

CA-SFM (2008). Comité de l'Antibiogramme de la Société Française de Microbiologie,
Recommandations 2008. Paris: Société Française de Microbiologie.

CLSI (2008). Performance Standards for Antimicrobial Susceptibility Testing, 18th informational supplement, M100-S18. Wayne, PA: Clinical and Laboratory Standards Institute.

Denapaite, D., Chi, F., Maurer, P., Nolte, O. \& Hakenbeck, R. (2007). Mechanism of penicillin resistance in Streptococcus pneumoniae: targets, gene transfer, and mutations. In Molecular Biology of Streptococci, pp. 290-303. Edited by R. Hakenbeck \& G. S. Chhatwal. Wymondham: Horizon Biosciences.

Guerin-Faublee, V., Tardy, F., Bouveron, C. \& Carret, G. (2002). Antimicrobial susceptibility of Streptococcus species isolated from clinical mastitis in dairy cows. Int $J$ Antimicrob Agents 19, 219-226.

Haenni, M., Galofaro, L., Ythier, M., Giddey, M., Majcherczyk, P., Moreillon, P. \& Madec, J.-Y. (2010). Penicillin-binding protein gene alterations in Streptococcus uberis isolates presenting decreased susceptibility to penicillin. Antimicrob Agents Chemother 54, 1140-1145.

Hoban, D., Baquero, F., Reed, V. \&

Felmingham, D. (2005). Demographic analysis of antimicrobial resistance among Streptococcus pneumoniae: worldwide results from PROTEKT 1999-2000. Int J Infect Dis 9, 262-273.

Kimura, K., Suzuki, S., Wachino, J., Kurokawa, H., Yamane, K., Shibata, N., Nagano, N., Kato, H., Shibayama, K. \& other authors (2008). First molecular characterization of group $B$ streptococci with reduced penicillin susceptibility. Antimicrob Agents Chemother 52, 2890-2897.
Mevius, D. J., Wit, I. B. \& Van Pelt, W. (editors) (2007). MARAN 2007 - Monitoring of Antimicrobial Resistance and Antibiotic Usage in Animals in The Netherlands in 2006/2007.

Lelystad: CVI-Lelystad.

Nagai, K., Davies, T. A., Jacobs, M. R. \& Appelbaum, P. C. (2002). Effects of amino acid alterations in penicillin-binding proteins (PBPs) 1a, 2b, and $2 x$ on PBP affinities of penicillin, ampicillin, amoxicillin, cefditoren, cefuroxime, cefprozil, and cefaclor in 18 clinical isolates of penicillin-susceptible, -intermediate, and -resistant pneumococci. Antimicrob Agents Chemother 46, 1273-1280.

Perez-Trallero, E., Fernandez-Mazarrasa, C. Garcia-Rey, C., Bouza, E., Aguilar, L., Garcia-de-Lomas, J. \& Baquero, F. (2001). Antimicrobial susceptibilities of 1,684 Streptococcus pneumoniae and 2,039 Streptococcus pyogenes isolates and their ecological relationships: results of a 1-year (1998-1999) multicenter surveillance study in Spain. Antimicrob Agents Chemother 45, 33343340.

Riffon, R., Sayasith, K., Khalil, H., Dubreuil, P., Drolet, M. \& Lagace, J. (2001)

Development of a rapid and sensitive test for identification of major pathogens in bovine mastitis by PCR. J Clin Microbiol 39, 25842589.

Rossitto, P. V., Ruiz, L., Kikuchi, Y., Glenn, K., Luiz, K., Watts, J. L. \& Cullor, J. S. (2002).

Antibiotic susceptibility patterns for environmental streptococci isolated from bovine mastitis in central California dairies. J Dairy Sci $85,132-138$. 